\title{
Solution of a diffusion problem in a non-homogeneous flow and diffusion field by the integral representation method (IRM)
}

\author{
Hiroshi Isshiki, Shuichi Nagata, Yasutaka Imai \\ Institute of Ocean Energy, Saga University, Saga, Japan \\ Email address: \\ isshiki@dab.hi-ho.ne.jp (H. Isshiki),nagata@ioes.saga-u.ac.jp (S. Nagata), imaiy@cc.saga-u.ac.jp (Y. Imai)
}

\section{To cite this article:}

Hiroshi Isshiki, Shuichi Nagata, Yasutaka Imai. Solution of a Diffusion Problem in a Non-Homogeneous Flow and Diffusion Field by the Integral Representation Method (IRM). Applied and Computational Mathematics. Vol. 3, No. 1, 2014, pp. 15-26.

doi: 10.11648/j.acm.20140301.13

\begin{abstract}
Integral representations are derived from a differential-type boundary value problem using a fundamental solution. A set of integral representations is equivalent to a set of differential equations. If the boundary conditions are substituted into the integral representations, the integral equations are obtained, and the unknown variables are determined by solving the integral equations. In other words, an integral-type boundary value problem is derived from the integral representations. An effective and flexible finite element algorithm is easily obtained from the integral-type boundary value problem. In the present paper, integral representations are obtained for the diffusion of a material or heat in the sea, where the convective velocity and diffusion constant change in space and time. A new numerical solution of an advection-diffusion equation is proposed based integral representations using the fundamental solution of the primary space-differential operator, and the numerical results are shown. An innovative generalization of the integral representation method: generalized integral representation method is also proposed. The numerical examples are given to verify the theory.
\end{abstract}

Keywords: Advection-Diffusion Problem, Variable Diffusion Constant, Integral Representation Method, Primary Space-Differential Operator, Generalized Fundamental Solution,

Generalized Integral Representation Method Component

\section{Introduction}

Generally speaking, physical phenomena are described as boundary value problems in differential equations. We refer to this type of problem as a differential-type boundary value problem. If we use a fundamental solution of the differential equations, we can derive integral representations from the differential-type boundary value problem. If we substitute the boundary conditions into the integral representations, we obtain the integral equations. We can determine the unknown variables by solving the integral equations. The integral representations are equivalent to the differential-type boundary value problem. Hence, we refer to the boundary value problem expressed by the integral representations as the integral-type boundary value problem.

If the diffusion coefficient is constant, a solution obtained by the boundary element method (BEM) is well known [1]. In the present paper, we discuss a solution obtained by the integral representation method (IRM) where the diffusion constant is not actually constant. In this case, a solution can be obtained using the finite element method (FEM) or the BEM with iteration (BEMI).

For the ordinary FEM algorithm of a boundary value problem, we must divide the computational region into elements and interpolate unknown functions. In the FEM, we use simple interpolation functions in the elements. This may reduce the degrees of freedom of the interpolation functions, and we overcome this difficulty by increasing the number of elements. As such, we face a serious problem in the mesh division. Although mesh-free methods [2 7] can be used to solve this problem, we encounter some difficulties in constructing the equilibrium equations of nodes and/or the stability of the calculation results. A collocation method such as that described in Reference [8] brings about difficulties in the construction of the interpolation function. On the other hand, the IRM can 
realize an easier division into elements and a higher precision interpolation. In the IRM, since the continuity of unknown variables between the elements is not required explicitly, a mesh-free approach would be possible in the case of a constant distribution of unknown variables in elements. If we introduce, for example, the moving least squares (MLS) method into the IRM, a mesh-free method would be feasible.

In the present paper, we propose a new numerical method for solving the diffusion equation:

(1) The proposed method is a unique numerical method hinted from integral representations of Navier-Stokes equation obtained by Uhlman [9].

(2) We can derive integral representations that do not include the differentiations of unknown variables with respect to spatial variables. We can easily introduce an irregular element division of the region. The present method would be favorable when the fluid region is geometrically complex and/or when the boundary changes in time and the element division inevitably becomes irregular.

(3) Since we need to consider only the fluid region in which the material or heat exists, the required calculation time and computer memory may be reduced.

We conduct numerical calculations of $1 \mathrm{D}$ and 2D problems and demonstrate the effectiveness of the IRM. Stable and precise results are obtained in a short time.

Furthermore, we developed a generalized integral representation method (GIRM). The integral representation based on the primary space-differentiation operator discussed above is one of the generalized integral representations (GIR). The primary space-differentiation operator is closely related to the differential operator of the boundary value-problem. On the other hand, in the generalized theory, the fundamental function is chosen first, and a differential equation is defined properly reflecting the fundamental solution and the boundary value problem. For example, we can use the Gaussian function as the generalized fundamental solution.

\section{Advection-Diffusion Equation in One-Dimension (1D)}

The spatial coordinate and time are denoted by $x$ and $t$, respectively. Let $C(x, t), u(x, t), \kappa(x, t)$ and $\sigma(x, t)$ be the material density or temperature, advective velocity, diffusion coefficient and material source, respectively. The $1 \mathrm{D}$ diffusion equation in region $0<x<L$ is then written as

$$
\begin{aligned}
\frac{\partial C}{\partial t}+u \frac{\partial C}{\partial x}=\frac{\partial}{\partial x}\left(\kappa \frac{\partial C}{\partial x}\right)+\sigma & =\kappa \frac{\partial^{2} C}{\partial x^{2}}+\frac{\partial \kappa}{\partial x} \frac{\partial C}{\partial x}+\sigma \\
\text { in } 0<x<L & <L
\end{aligned}
$$

If $C(x, t)$ at an internal point (IP) is known and $C(x, t)$ or $\partial C(x, t) / \partial x$ at a boundary point (BP) is given at time $t$ from the boundary conditions $(\mathrm{BC})$, then differential equation, (1), yields $\partial C(x, t) / \partial t$ at IP. $C(x, t+d t)$ at IP is then obtained by $C(x, t+d t)=C(x, t)+d t \partial C(x, t) / \partial t$. Hence, if $C(x, 0)$ is given by the initial condition, we can solve the initial-boundary value problem.

Rewriting (1), we have

$$
\frac{\partial^{2} C}{\partial x^{2}}=\frac{1}{\kappa}\left(\frac{\partial C}{\partial t}+u \frac{\partial C}{\partial x}-\frac{\partial \kappa}{\partial x} \frac{\partial C}{\partial x}-\sigma\right)
$$

We define the space fundamental solution $G(x, \xi)$ of (2) as

$$
\frac{\partial^{2} G(x, \xi)}{\partial x^{2}}=\delta(x, \xi)
$$

where $\delta(x, \xi)$ is Dirac's delta function $\delta(x-\xi)$ :

$$
\delta(x)=0 \text { for } x \neq 0 \text { and } \int_{-\infty}^{\infty} \delta(x) d x=1
$$

We refer to the operator $\partial^{2} / \partial x^{2}$ as the primary space-differential operator. Then, we have

$$
G(x, \xi)=\frac{1}{2}|x-\xi|
$$

The fundamental solution $G(x, \xi)$ satisfies

$$
\frac{\partial G(x, \xi)}{\partial x}=-\frac{\partial G(x, \xi)}{\partial \xi}=\frac{1}{2} \operatorname{sgn}(x-\xi) .
$$

From (2), we have

$$
\begin{aligned}
& 0=\int_{0}^{L}\left\{G ( x , \xi ) \left[\frac{\partial^{2} C(x, t)}{\partial x^{2}}\right.\right. \\
& \left.-\frac{1}{\kappa(x, t)}\left(\frac{\partial C(x, t)}{\partial t}+u(x, t) \frac{\partial C(x, t)}{\partial x}-\frac{\partial \kappa(x, t)}{\partial x} \frac{\partial C(x, t)}{\partial x}-\sigma(x, t)\right)\right] \\
& \left.-C(x, t)\left[\frac{\partial^{2} G(x, \xi)}{\partial x^{2}}-\delta(x, \xi)\right]\right\} d x
\end{aligned}
$$

Hence, we obtain

$$
\begin{aligned}
\varepsilon(\xi) C(\xi, t)= & -\int_{0}^{L}\left(G(x, \xi) \frac{\partial^{2} C(x, t)}{\partial x^{2}}-C(x, t) \frac{\partial^{2} G(x, \xi)}{\partial x^{2}}\right) d x \\
& +\int_{0}^{L} \frac{G(x, \xi)}{\kappa(x, t)}\left(\frac{\partial C(x, t)}{\partial t}+u(x, t) \frac{\partial C(x, t)}{\partial x}\right. \\
& \left.-\frac{\partial \kappa(x, t)}{\partial x} \frac{\partial C(x, t)}{\partial x}-\sigma(x, t)\right) d x
\end{aligned}
$$

where

$$
\varepsilon(\xi)=\left\{\begin{array}{l}
1 \quad \text { when } 0<x<L \\
1 / 2 \text { when } x=0 \text { or } \mathrm{x}=\mathrm{L} . \\
0 \quad \text { otherwise }
\end{array}\right.
$$

Transforming the integral of the first term on the right-hand side of (8) and exchanging $x$ and $\xi$, we obtain 


$$
\begin{aligned}
& \varepsilon(x) C(x, t)=-\left[G(x, \xi) \frac{\partial C(\xi, t)}{\partial \xi}-C(\xi, t) \frac{\partial G(x, \xi)}{\partial \xi}\right]_{\xi=0}^{\xi=L} \\
& +\int_{0}^{L} \frac{G(x, \xi)}{\kappa(\xi, t)} \frac{\partial C(\xi, t)}{\partial t} d \xi+\int_{0}^{L} \frac{G(x, \xi)}{\kappa(\xi, t)} u(\xi, t) \frac{\partial C(\xi, t)}{\partial \xi} d \xi \\
& -\int_{0}^{L} \frac{G(x, \xi)}{\kappa(\xi, t)} \frac{\partial \kappa(\xi, t)}{\partial \xi} \frac{\partial C(\xi, t)}{\partial \xi} d \xi-\int_{0}^{L} \frac{G(x, \xi)}{\kappa(\xi, t)} \sigma(\xi, t) d \xi
\end{aligned}
$$

If we remove the spatial derivatives of $C$ in the integrals in (10), we obtain

$$
\begin{aligned}
& \varepsilon(x) C(x, t)=-\left[G(x, \xi) \frac{\partial C(\xi, t)}{\partial \xi}-C(\xi, t) \frac{\partial G(x, \xi)}{\partial \xi}\right]_{\xi=0}^{\xi=L} \\
& +\left[\frac{G(x, \xi)}{\kappa(\xi, t)} u(\xi, t) C(\xi, t)\right]_{\xi=0}^{\xi=L}-\left[\frac{G(x, \xi)}{\kappa(\xi, t)} \frac{\partial \kappa(\xi, t)}{\partial \xi} C(\xi, t)\right]_{\xi=0}^{\xi=L} \\
& +\int_{0}^{L} \frac{G(x, \xi)}{\kappa(\xi, t)} \frac{\partial C(\xi, t)}{\partial t} d \xi-\int_{0}^{L} C(\xi, t) \frac{\partial}{\partial \xi}\left(\frac{G(x, \xi)}{\kappa(\xi, t)} u(\xi, t)\right) d \xi \\
& +\int_{0}^{L} C(\xi, t) \frac{\partial}{\partial \xi}\left(\frac{G(x, \xi)}{\kappa(\xi, t)} \frac{\partial \kappa(\xi, t)}{\partial \xi}\right) d \xi-\int_{0}^{L} \frac{G(x, \xi)}{\kappa(\xi, t)} \sigma(\xi, t) d \xi
\end{aligned}
$$

If $C(x, t)$ at IP is known and $C(x, t)$ or $\partial C(x, t) / \partial x$ at $\mathrm{BP}$ is known from the boundary conditions, then the integral representations are integral equations with $\partial C(x, t) / \partial t$ the unknown variables at IP and $\partial C(x, t) / \partial x$ or $C(x, t)$ the unknown variables at BP. We obtain $C(x, t+d t)$ at IP from $C(x, t+d t)=C(x, t)+d t \partial C(x, t) / \partial t$. If $C(x, 0)$ on IP is known from the initial conditions, then we can solve the initial and boundary value problem using the integral representations.

When the diffusion coefficient $\kappa(x, t)$ and the advective velocity $u(x, t)$ are given simply by

$$
\begin{aligned}
& \kappa(x, t)=\kappa=\text { const }, \\
& u(x, t)=U=\text { const },
\end{aligned}
$$

and (5) and (6) are substituted into (11), we obtain

$$
\begin{aligned}
& \varepsilon(x) C(x, t)=\frac{1}{2 \kappa} \int_{0}^{L}|x-\xi| \frac{\partial C(\xi, t)}{\partial t} d \xi+\frac{U}{2 \kappa} \int_{0}^{L} C(\xi, t) \operatorname{sgn}(x-\xi) d \xi \\
& -\frac{1}{2 \kappa} \int_{0}^{L}|x-\xi| \sigma(\xi, t) d \xi+\frac{U}{2 \kappa}|x-L| C(L, t)-\frac{U}{2 \kappa}|x| C(0, t) \\
& -\frac{1}{2}|x-L| \frac{\partial C(L, t)}{\partial x}+\frac{1}{2}|x| \frac{\partial C(0, t)}{\partial x} \\
& -\frac{1}{2} C(L, t) \operatorname{sgn}(x-L)+\frac{1}{2} C(0, t) \operatorname{sgn}(x)
\end{aligned}
$$

\section{Numerical Applications to One-Dimensional (1D) Problems}

For simplicity, we assume the conditions in (12) and $\sigma=0$. First, we transform the integral representation, (13), into algebraic equations. We divide the region $0 \leq x \leq L$ into $N$ equal elements of length $d x$ and denote the midpoint of each element as $x_{i}, \quad i=0,1, \cdots, N-1$ as follows:

$$
d x=\frac{L}{N}
$$

and

$$
x_{i}=(0.5+i) d x \text {. }
$$

Equation (13) is approximated by

$$
\begin{aligned}
\mathcal{E}(x) C(x, t)= & \frac{1}{2 \kappa} \sum_{j=0}^{N-1} \frac{\partial C\left(x_{j}, t\right)}{\partial t} \int_{x_{j}-d x / 2}^{x_{j}-d x / 2}|x-\xi| d \xi \\
& +\frac{U}{2 \kappa} \sum_{j=0}^{N-1} C\left(x_{j}, t\right) \int_{x_{j}-d x / 2}^{x_{j}-d x / 2} \operatorname{sgn}(x-\xi) d \xi \\
& +\frac{U}{2 \kappa}|x-L| C(L, t)-\frac{U}{2 \kappa}|x| C(0, t) \\
& +\frac{1}{2} \operatorname{sgn}(L-x) C(L, t)+\frac{1}{2} \operatorname{sgn}(x) C(0, t) \\
& -\frac{1}{2} \frac{\partial C(L, t)}{\partial x}|L-x|+\frac{1}{2} \frac{\partial C(0, t)}{\partial x}|x|
\end{aligned}
$$

We define $\alpha_{i j}$ for the internal point (IP) $x=x_{i}$ and $\alpha_{B P j}$ for the boundary point (BP) $x=0$ or $x=L$ as

$$
\begin{gathered}
\alpha_{i j}=\int_{x_{j}-d x / 2}^{x_{j}-d x / 2}\left|x_{i}-\xi\right| d \xi= \begin{cases}d x^{2} / 4 & \text { when } i=j \\
\left|x_{i}-x_{j}\right| d x & \text { when } i \neq j\end{cases} \\
\alpha_{B P j}=\int_{x_{j}-d x / 2}^{x_{j}+d x / 2}|x-\xi| d \xi=\left|x-x_{j}\right| d x
\end{gathered}
$$

Then we obtain the algebraic equations for IP ( $x_{i}$, $i=0,1, \cdots, N-1)$ and $\mathrm{BP}\left(x_{B P}=0, L\right)$, respectively, as

$$
\begin{aligned}
C\left(x_{i}, t\right)= & \frac{1}{2 \kappa} \sum_{j=0}^{N-1} \alpha_{i j} \frac{\partial C\left(x_{j}, t\right)}{\partial t} \\
& +\frac{U}{2 \kappa} \sum_{j=0}^{N-1} \int_{x_{j}-d x / 2}^{x_{j}-d x / 2} \operatorname{sgn}\left(x_{i}-\xi\right) d \xi C\left(x_{j}, t\right) \\
& +\frac{U}{2 \kappa}\left|x_{i}-L\right| C(L, t)-\frac{U}{2 \kappa}\left|x_{i}\right| C(0, t) \\
& +\frac{1}{2} \operatorname{sgn}(L-x) C(L, t)+\frac{1}{2} \operatorname{sgn}(x) C(0, t) \\
& -\frac{1}{2}\left|L-x_{i}\right| \frac{\partial C(L, t)}{\partial x}+\frac{1}{2}\left|x_{i}\right| \frac{\partial C(0, t)}{\partial x} \\
\frac{1}{2} C\left(x_{B P}, t\right)= & \frac{1}{2 \kappa} \sum_{j=0}^{N-1} \alpha_{B P j} \frac{\partial C\left(x_{j}, t\right)}{\partial t} \\
& +\frac{U}{2 \kappa} \sum_{j=0}^{N-1} \operatorname{sgn}\left(x_{B P}-\xi\right) d \xi C\left(x_{j}, t\right) \\
& +\frac{U}{2 \kappa}\left|x_{B P}-L\right| C(L, t)-\frac{U}{2 \kappa}\left|x_{B P}\right| C(0, t) \\
& +\frac{1}{2} \operatorname{sgn}\left(L-x_{B P}\right) C(L, t)+\frac{1}{2} \operatorname{sgn}\left(x_{B P}\right) C(0, t) \\
& -\frac{1}{2}\left|L-x_{B P}\right| \frac{\partial C(L, t)}{\partial x}+\frac{1}{2}\left|x_{B P}\right| \frac{\partial C(0, t)}{\partial x}
\end{aligned}
$$

There are two types of solution of these algebraic equations, (18): explicit and implicit solutions.

\section{Explicit solution A1}

In the integral representation, $(18), \partial C(x, t) / \partial t$ is used as the main unknown variable. 
(1) Assume $C(x, t)$ known.

(2) Obtain $\partial C(x, t) / \partial t$ using (18).

(3) Obtain $C(x, t+d t)$ from $C(x, t+d t)=C(x, t)+(\partial C(x, t) / \partial t) d t$.

(4) Repeat the process.

\section{Implicit solution $\boldsymbol{A 2}$}

In the integral representation, (18), $C(x, t)$ is used as the main unknown variable.

(1) Assume $C(x, t-d t)$ known.

(2) Approximate $\partial C(x, t) / \partial t$ in (18) by $\partial C(x, t) / \partial t=[C(x, t)-C(x, t-d t)] / d t$ and solve for the unknown variable $C(x, t)$.

(3) Repeat the process.

If we use implicit solution, the stability of the numerical calculation increases much, and we can use much larger $d t$ than the one used in the explicit method. The non-homogeneous term of the integral equation must be evaluated at $t-0.5 d t$.

In the case of explicit solution A1, we use the following algebraic equations and progression of time equation:

$$
\begin{aligned}
& C\left(x_{i}, t\right)-\frac{U}{2 \kappa}\left|x_{i}-L\right| C(L, t)+\frac{U}{2 \kappa}\left|x_{i}\right| C(0, t) \\
& -\frac{1}{2} \operatorname{sgn}\left(L-x_{i}\right) C(L, t)-\frac{1}{2} \operatorname{sgn}\left(x_{i}\right) C(0, t) \\
& +\frac{1}{2} \frac{\partial C(L, t)}{\partial x}\left|L-x_{i}\right|-\frac{1}{2} \frac{\partial C(0, t)}{\partial x}\left|x_{i}\right| \\
& \quad-\frac{U}{2 \kappa} \sum_{j=0}^{N-1} \int_{x_{j}-d x / 2}^{x_{j}-d x / 2} \operatorname{sgn}\left(x_{i}-\xi\right) d \xi C\left(x_{j}, t\right)=\frac{1}{2 \kappa} \sum_{j=0}^{N-1} \alpha_{i j} \frac{\partial C\left(x_{j}, t\right)}{\partial t} \\
& \frac{1}{2} C\left(x_{B P}, t\right)-\frac{U}{2 \kappa}\left|x_{B P}-L\right| C(L, t)+\frac{U}{2 \kappa}\left|x_{B P}\right| C(0, t) \\
& -\frac{1}{2} \operatorname{sgn}\left(L-x_{B P}\right) C(L, t)-\frac{1}{2} \operatorname{sgn}\left(x_{B P}\right) C(0, t) \\
& +\frac{1}{2} \frac{\partial C(L, t)}{\partial x}\left|L-x_{B P}\right|-\frac{1}{2} \frac{\partial C(0, t)}{\partial x}\left|x_{B P}\right| \\
& -\frac{U}{2 \kappa} \sum_{j=0}^{N-1} \int_{x_{j}-d x / 2}^{x_{j}-d x / 2} \operatorname{sgn}\left(x_{B P}-\xi\right) d \xi C\left(x_{j}, t\right)=\frac{1}{2 \kappa} \sum_{j=0}^{N-1} \alpha_{i j} \frac{\partial C\left(x_{j}, t\right)}{\partial t} \\
& C\left(x_{i}, t+d t\right)=C\left(x_{i}, t\right)+\frac{\partial C\left(x_{i}, t\right)}{\partial t} d t .
\end{aligned}
$$

The total number of the unknowns in the algebraic equations is $N+2:$ where $\partial C\left(x_{j}, t\right) / \partial t$ for $j=0,1, \cdots, N-1$, and $C(0, t)$ or $\partial C(0, t) / \partial x$ and $C(L, t)$ or $\partial C(L, t) / \partial x$. The total number of equations is also $N+2$ in total: $N$ equations for IP and 2 equations for BP.

In this case, all variables on IP and BP become unknowns. Namely, we are facing a region-boundary element problem. As an approximation, we use $\partial C(0, t) / \partial y \approx 2\left[C\left(x_{0}, t\right)-C(0, t)\right] / d x$, if $C(0, t)$ is known. Otherwise, we use $C(0, t) \approx C\left(x_{0}, t\right)-[\partial C(0, t) / \partial x] \cdot d x / 2$, if $\partial C(0, t) / \partial x$ is known. We use similar approximations for $x=L$. In this case, the number of unknowns and the number of equations are both $N$.

In solution $\mathrm{A} 2$, we can obtain the similar algebraic equations as in solution A1.

\subsection{Diffusion of Material in Infinite Space without Advection}

We consider an initial value problem without advection in the region $-\infty<x<\infty \quad(-L<x<L$ in computation) with the initial condition $C(x, 0)=\delta(x)$. As a result of the symmetry, we consider the region given by $0 \leq x<\infty$ $(0<x<L$ in computation). We summarize the conditions as follows:

Initial condition:

$$
C\left(x_{i}, 0\right)=\frac{1}{2 d x} \delta_{i 0}, \quad i=0,1, \cdots, N-1,
$$

where $\delta_{i j}$ is the Kronecker delta: $\delta_{i j}$ is 1 if $i=j$ or 0 otherwise.

Boundary condition:

$$
\begin{gathered}
\frac{\partial C(0, t)}{\partial x}=0, C(L, t)=0, \frac{\partial C(L, t)}{\partial x}=0 \\
\text { Approximation : } C(0, t) \approx C\left(x_{0}, t\right)
\end{gathered}
$$

In the case of procedure A1, we solve from (19) with $U=0$

$$
\begin{array}{r}
2 \kappa\left[C\left(x_{i}, t\right)-\frac{1}{2} C\left(x_{0}, t\right)\right]=\sum_{j=0}^{N-1} \alpha_{i j} \frac{\partial C\left(x_{j}, t\right)}{\partial t}, \\
i=0,1, \cdots, N-1, \\
C\left(x_{i}, t+d t\right)=C\left(x_{i}, t\right)+\frac{\partial C\left(x_{i}, t\right)}{\partial t} d t, \\
i=0,1, \cdots, N-1 .
\end{array}
$$

The solution of this problem is the well-known fundamental solution of the linear 1D diffusion problem:

$$
C(x, t)=\frac{1}{2 \sqrt{\pi \nu t}} e^{-\frac{x^{2}}{4 \kappa t}} .
$$

Numerical results are shown in Figs. 1 and 2. We used procedure A1 for the numerical calculation. The calculation conditions are $L=1, N=20, d t=0.0005$ and $v=0.089$. The precision of the calculation is very high.

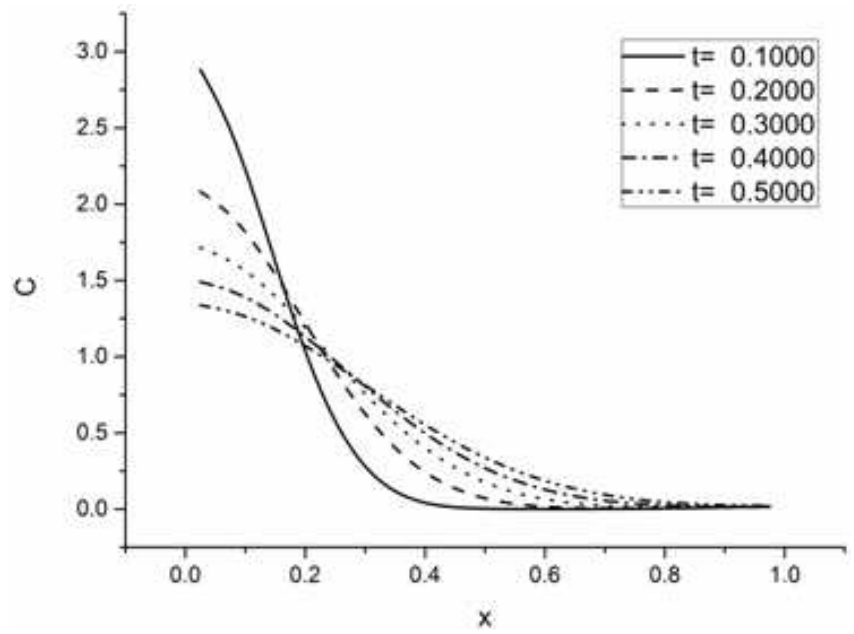

Figure 1. Calculated results at each time step. 


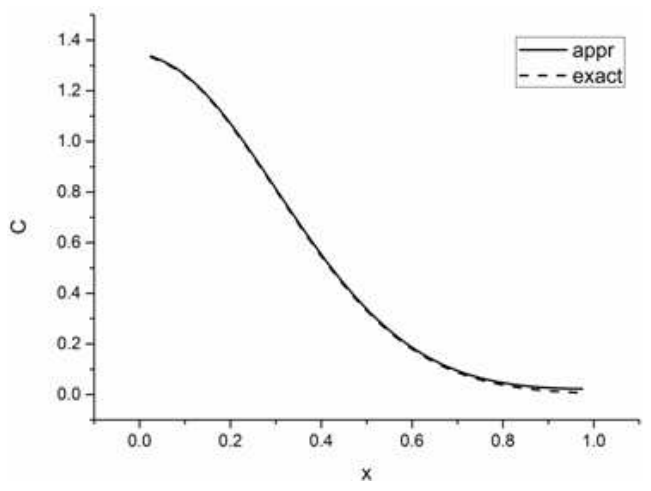

Figure 2. Comparison with the exact solution $(t=0.5)$.

\subsection{Diffusion of Material in Infinite Space with Advection}

In the calculations in Section 3.1, we considered in the region given by $0 \leq x<\infty \quad(0 \leq x<L$ in computation) using symmetry. However, since this problem is asymmetric, we must consider in the region given by $-\infty<x<\infty$ ( $-L \leq x<L$ in computation). When $-L \leq x<L$ is large enough, we can assume

$$
\frac{\partial C( \pm L, t)}{\partial x} \approx 0, C( \pm L, t) \approx 0
$$

If we consider a doublet-type initial distribution of material, the initial condition is given by

$$
C(x, 0) \approx \frac{-\delta(x-d x / 2)+\delta(x+d x / 2)}{d x}
$$

In this case, the size and center of elements are given by

$$
\begin{gathered}
d x=\frac{2 L}{N}, \\
x_{i}=-L+(0.5+i) d x, \quad i=0,1, \cdots, N-1 .
\end{gathered}
$$

From (26), the initial condition in this case is specifically as follows:

$$
C\left(x_{i}, 0\right)=-\frac{1}{d x^{2}} \delta_{i N / 2}+\frac{1}{d x^{2}} \delta_{i N / 2+1}, \quad i=0,1, \cdots, N-1,
$$

since

$$
\begin{gathered}
\delta(x-d x / 2) \approx\left\{\begin{array}{ll}
1 / d x & \text { in } x_{N / 2}-d x / 2<x<x_{N / 2}+d x / 2 \\
0 & \text { otherwise }
\end{array},\right. \\
\delta(x+d x / 2) \approx \begin{cases}1 / d x & \text { in } x_{N / 2+1}-d x / 2<x<x_{N / 2+1}+d x / 2 \\
0 & \text { otherwise }\end{cases}
\end{gathered}
$$

If we adopt the explicit solution A1, we have from (19)

$$
\begin{aligned}
2 \kappa C\left(x_{i}, t\right)-U \sum_{j=0}^{N-1} \operatorname{sgn}\left(x_{i}-x_{j}\right) d x C\left(x_{j}, t\right) & =\sum_{j=0}^{N-1} \alpha_{i j} \frac{\partial C\left(x_{j}, t\right)}{\partial t}, \\
i & =0,1, \cdots, N-1 .
\end{aligned}
$$

For the check of the calculation, we use

$$
\begin{aligned}
\int_{-\infty}^{\infty} \frac{\partial C(x, t)}{\partial t} d x & =\int_{-\infty}^{\infty}\left[-U \frac{\partial C(x, t)}{\partial x}+\kappa \frac{\partial^{2} C(x, t)}{\partial x^{2}}\right] d x \\
& =\left[-U C(x, t)+\kappa \frac{\partial C(x, t)}{\partial x}\right]_{x=-\infty}^{x=\infty}=0
\end{aligned}
$$

Hence, we have

$$
\int_{-\infty}^{\infty} C(x, t) d x=\text { const } .
$$

The exact solution of the problem is given by

$$
C(x, t)=\frac{1}{2 \sqrt{\pi \kappa t}} \frac{x-U t}{2 \kappa t} e^{-\frac{(x-U t)^{2}}{4 k t}} .
$$

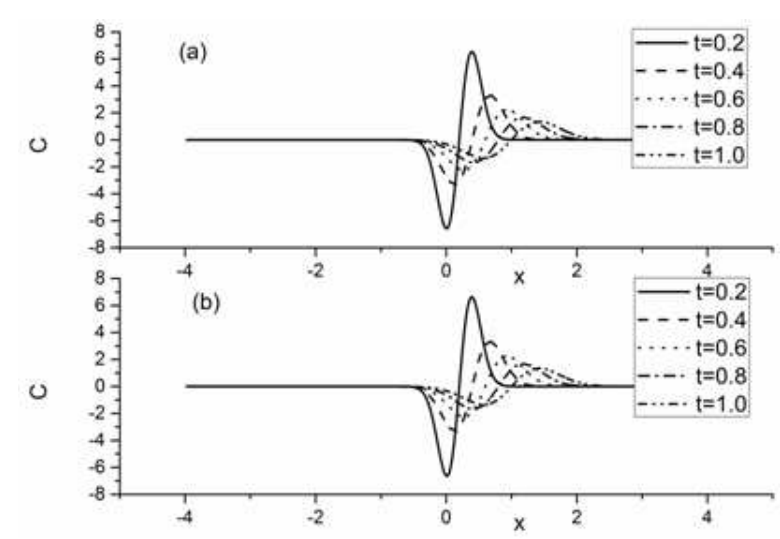

Figure 3. Space distribution of $C$ in case of doublet type initial distribution of material: (a) approximate solution; (b) exact solution.(48)

Numerical results are shown in Fig. 3. We used A1 procedure for the numerical calculation. The calculation conditions are $L=4, N=160, d t=0.0005, \kappa=0.089$ and $U=1.0$. The precision of the calculation is high.

\section{Advection-Diffusion Equation in Two-Dimension (2D)}

The spatial coordinate and time are denoted as $(x, y)$ and $t$, respectively. Let $C(x, y, t), \mathbf{u}(x, y, t)=u(x, y, t) \mathbf{i}+v(x, y, t) \mathbf{j}$, $\kappa(x, y, t)$, and $\sigma(x, y, t)$ be the material density or temperature, the advective velocity vector, the diffusion coefficient, and the material source, respectively. The 2D diffusion equation in region $S$ is then written as

$$
\frac{\partial C}{\partial t}+(\mathbf{u} \cdot \nabla) C=\nabla \cdot(\kappa \nabla C)+\sigma=\kappa \nabla^{2} C+\nabla \kappa \cdot \nabla C+\sigma
$$

in $S$

Rewriting (34), we have

$$
\nabla^{2} C=\frac{1}{\kappa}\left(\frac{\partial C}{\partial t}+(\mathbf{u} \cdot \nabla) C-\nabla \kappa \cdot \nabla C-\sigma\right) .
$$

The fundamental solution of Laplace operator, the primary space-differential operator in this case, is defined as 


$$
\nabla^{2} G(\mathbf{x}, \xi)=\delta(\mathbf{x}, \xi)
$$

and is given by

$$
G(\mathbf{x}, \xi)=\frac{1}{2 \pi} \ln r
$$

where $\delta(\mathbf{x}, \boldsymbol{\xi})=\delta(x-\xi) \delta(y-\eta)$ and $r=|\mathbf{x}-\xi|=\sqrt{(x-\xi)^{2}+(y-\eta)^{2}}$. The fundamental solution $G(\mathbf{x}, \xi)$ satisfies

$$
\nabla_{\mathbf{x}} G(\mathbf{x}, \xi)=-\nabla_{\xi} G(\mathbf{x}, \xi)=\frac{1}{2 \pi} \frac{\mathbf{r}}{r^{2}}=\frac{1}{2 \pi} \frac{(\mathbf{x}-\xi)}{|\mathbf{x}-\xi|^{2}}
$$

An extension to 3D may be straight forward. Let $r$ in 3D be $\quad r=|\mathbf{x}-\boldsymbol{\xi}|=\sqrt{(x-\xi)^{2}+(y-\eta)^{2}+(z-\zeta)^{2}}$ and the fundamental solution be

$$
\begin{gathered}
G(\mathbf{x}, \xi)=-\frac{1}{4 \pi r}, \\
\nabla_{\mathbf{x}} G(\mathbf{x}, \xi)=-\nabla_{\xi} G(\mathbf{x}, \xi)=\frac{1}{4 \pi} \frac{\mathbf{r}}{r^{3}}=\frac{1}{4 \pi} \frac{(\mathbf{x}-\xi)}{|\mathbf{x}-\xi|^{3}} .
\end{gathered}
$$

And we replace area and line integrals by volume and area integrals, respectively.

From (35) and (36), we have

$$
\begin{aligned}
0 & =\iint_{S}\left\{G ( \mathbf { x } , \xi ) \left[\nabla_{\mathbf{x}}^{2} C(\mathbf{x}, t)\right.\right. \\
& -\frac{1}{\kappa(\mathbf{x}, t)}\left(\frac{\partial C(\mathbf{x}, t)}{\partial t}+\left(\mathbf{u}(\mathbf{x}, t) \cdot \nabla_{\mathbf{x}}\right) C(\mathbf{x}, t)\right. \\
& \left.\left.-\nabla_{\mathbf{x}} \kappa(\mathbf{x}, t) \cdot \nabla_{\mathbf{x}} C(\mathbf{x}, t)-\sigma(\mathbf{x}, t)\right)\right] \\
& \left.-C(\mathbf{x}, t)\left[\nabla_{\mathbf{x}}^{2} G(\mathbf{x}, \xi)-\delta(\mathbf{x}, \xi)\right]\right\} d S_{\mathbf{x}}
\end{aligned}
$$

Hence, we obtain

$$
\begin{aligned}
& \varepsilon(\xi) C(\boldsymbol{\xi}, t)=-\iint_{S}\left(G(\mathbf{x}, \xi) \nabla_{\mathbf{x}}^{2} C(\mathbf{x}, t)-C(\mathbf{x}, t) \nabla_{\mathbf{x}}^{2} G(\mathbf{x}, \xi)\right) d S_{\mathbf{x}} \\
& +\iint_{S} \frac{G(\mathbf{x}, \boldsymbol{\xi})}{\kappa(\mathbf{x}, t)}\left(\frac{\partial C(\mathbf{x}, t)}{\partial t}+\left(\mathbf{u}(\mathbf{x}, t) \cdot \nabla_{\mathbf{x}}\right) C(\mathbf{x}, t)\right. \\
& \left.-\nabla_{\mathbf{x}} \kappa(\mathbf{x}, t) \cdot \nabla_{\mathbf{x}} C(\mathbf{x}, t)-\sigma(\mathbf{x}, t)\right) d S_{\mathbf{x}}
\end{aligned}
$$

where

$$
\varepsilon(\xi)=\left\{\begin{array}{l}
1 \quad \text { when } \xi \in S \\
1 / 2 \quad \text { when } \xi \in \bar{C}, \\
0 \quad \text { otherwise }
\end{array}\right.
$$

and $\bar{C}$ is the boundary of $S$.

We transform the integral of the first term on the right-hand side of (41). Using the vector formula:

$$
\begin{aligned}
& G(\mathbf{x}, \xi) \nabla_{\mathbf{x}}^{2} C(\mathbf{x}, t)-C(\mathbf{x}, t) \nabla_{\mathbf{x}}^{2} G(\mathbf{x}, \xi) \\
& =\nabla_{\mathbf{x}} \cdot\left(G(\mathbf{x}, \xi) \nabla_{\mathbf{x}} C(\mathbf{x}, t)\right)-\nabla_{\mathbf{x}} \cdot\left(C(\mathbf{x}, t) \nabla_{\mathbf{x}} G(\mathbf{x}, \xi)\right)
\end{aligned}
$$

we obtain

$$
\begin{aligned}
& \varepsilon(\xi) C(\xi, t)=-\int_{\bar{C}}\left(G(\mathbf{x}, \xi) \frac{\partial C(\mathbf{x}, t)}{\partial n_{\mathbf{x}}}-C(\mathbf{x}, t) \frac{\partial G(\mathbf{x}, \boldsymbol{\xi})}{\partial n_{\mathbf{x}}}\right) d l_{\mathbf{x}} \\
& +\iint_{S} \frac{G(\mathbf{x}, \boldsymbol{\xi})}{\kappa(\mathbf{x}, t)} \frac{\partial C(\mathbf{x}, t)}{\partial t} d S_{\mathbf{x}}+\iint_{S} \frac{G(\mathbf{x}, \boldsymbol{\xi})}{\kappa(\mathbf{x}, t)}\left(\mathbf{u}(\mathbf{x}, t) \cdot \nabla_{\mathbf{x}}\right) C(\mathbf{x}, t) d S_{\mathbf{x}} \\
& -\iint_{S} \frac{G(\mathbf{x}, \boldsymbol{\xi})}{\kappa(\mathbf{x}, t)} \nabla_{\mathbf{x}} \kappa(\mathbf{x}, t) \cdot \nabla_{\mathbf{x}} C(\mathbf{x}, t) d S_{\mathbf{x}}-\iint_{S} \frac{G(\mathbf{x}, \boldsymbol{\xi})}{\kappa(\mathbf{x}, t)} \sigma(\mathbf{x}, t) d S_{\mathbf{x}}
\end{aligned}
$$

where $\mathbf{n}$ is the unit outward normal on $\bar{C}$. Exchanging $\mathbf{x}$ and $\xi$, we have

$$
\begin{aligned}
& \varepsilon(\mathbf{x}) C(\mathbf{x}, t)=-\int_{\bar{C}}\left(G(\mathbf{x}, \xi) \frac{\partial C(\xi, t)}{\partial n_{\xi}}-C(\xi, t) \frac{\partial G(\mathbf{x}, \xi)}{\partial n_{\xi}}\right) d l_{\xi} \\
& +\iint_{S} \frac{G(\mathbf{x}, \xi)}{\kappa(\xi, t)} \frac{\partial C(\xi, t)}{\partial t} d S_{\xi}+\iint_{S} \frac{G(\mathbf{x}, \xi)}{\kappa(\xi, t)}\left(\mathbf{u}(\xi, t) \cdot \nabla_{\xi}\right) C(\xi, t) d S_{\xi} \\
& -\iint_{S} \frac{G(\mathbf{x}, \xi)}{\kappa(\xi, t)} \nabla_{\xi} \kappa(\xi, t) \cdot \nabla_{\xi} C(\xi, t) d S_{\xi}-\iint_{S} \frac{G(\mathbf{x}, \xi)}{\kappa(\xi, t)} \sigma(\xi, t) d S_{\xi}
\end{aligned}
$$

Removing the spatial derivatives of $C(\mathbf{x}, t)$ in the area integrals in (45), we have

$$
\begin{aligned}
\varepsilon(\mathbf{x}) C(\mathbf{x}, t)= & -\int_{\bar{C}}\left(G(\mathbf{x}, \xi) \frac{\partial C(\xi, t)}{\partial n_{\xi}}-C(\xi, t) \frac{\partial G(\mathbf{x}, \xi)}{\partial n_{\xi}}\right) d l_{\xi} \\
& +\int_{\bar{C}} \frac{G(\mathbf{x}, \xi)}{\kappa(\mathbf{x}, \xi)} C(\xi, t) \mathbf{u}(\xi, t) \cdot \mathbf{n} d l_{\xi}-\int_{\bar{C}} \frac{G(\mathbf{x}, \xi)}{\kappa(\xi, t)} \frac{\partial \kappa(\xi, t)}{\partial n_{\xi}} C(\xi, t) d l_{\xi} \\
& +\iint_{S} \frac{G(\mathbf{x}, \xi)}{\kappa(\xi, t)} \frac{\partial C(\xi, t)}{\partial t} d S_{\xi}-\iint_{S} C(\xi, t) \nabla_{\xi} \cdot\left(\frac{G(\mathbf{x}, \boldsymbol{\xi})}{\kappa(\mathbf{x}, \boldsymbol{\xi})} \mathbf{u}(\xi, t)\right) d S_{\xi} \\
& +\iint_{S} C(\xi, t) \nabla_{\xi} \cdot\left(\frac{G(\mathbf{x}, \xi)}{\kappa(\xi, t)} \nabla_{\xi} \kappa(\xi, t)\right) d S_{\xi}-\iint_{S} \frac{G(\mathbf{x}, \xi)}{\kappa(\xi, t)} \sigma(\xi, t) d S_{\xi}
\end{aligned}
$$

This equation does not include the spatial derivative of the unknown variable $C(\mathbf{x}, t)$ in the area integrals.

If $C(\mathbf{x}, t)$ at IP and $C(\mathbf{x}, t)$ or $\partial C(\mathbf{x}, t) / \partial n$ at $\mathrm{BP}$ are known from the boundary conditions, the integral representations are integral equations having unknown variables $\partial C(\mathbf{x}, t) / \partial t$ at IP and $\partial C(\mathbf{x}, t) / \partial n$ or $C(\mathbf{x}, t)$ at BP. We obtain $C(\mathbf{x}, t+d t)$ at IP from $C(\mathbf{x}, t+d t)=C(\mathbf{x}, t)+d t \partial C(\mathbf{x}, t) / \partial t$. Specifically, if $C(\mathbf{x}, 0)$ at IP is known from the initial conditions, we can solve the initial and boundary value problems using the integral representations.

When the diffusion coefficient $\kappa(\mathbf{x}, t)$ and the advective velocity $\mathbf{u}(\mathbf{x}, t)=u(\mathbf{x}, t) \mathbf{i}+v(\mathbf{x}, t) \mathbf{j}$ are given simply by

$$
\begin{aligned}
& \kappa(\mathbf{x}, t)=\kappa=\text { const }, \\
& \mathbf{u}(\mathbf{x}, t)=U \mathbf{i}=\text { const },
\end{aligned}
$$

and (37) and (38) are substituted into (46), we obtain

$$
\begin{aligned}
& \mathcal{E}(\mathbf{x}) C(\mathbf{x}, t)=\frac{1}{2 \pi \kappa} \iint_{S} \ln (|\mathbf{x}-\xi|) \frac{\partial C(\xi, t)}{\partial t} d S_{\xi} \\
& -\frac{U}{2 \pi \kappa} \iint_{S} C(\xi, t) \frac{\partial \ln (|\mathbf{x}-\xi|)}{\partial \xi} d S_{\xi} \\
& +\frac{U}{2 \pi \kappa} \int_{\bar{C}} \ln (|\mathbf{x}-\xi|) C(\xi, t) n_{\xi} d l_{\xi}-\frac{1}{2 \pi \kappa} \iint_{S} \ln (|\mathbf{x}-\xi|) \sigma(\xi, t) d S_{\xi} \\
& -\frac{1}{2 \pi} \int_{\bar{C}} \ln (|\mathbf{x}-\xi|) \frac{\partial C(\xi, t)}{\partial n_{\xi}} d l_{\xi}+\frac{1}{2 \pi} \int_{\bar{C}} \frac{\partial \ln (|\mathbf{x}-\boldsymbol{\xi}|)}{\partial n_{\xi}} C(\xi, t) d l_{\xi}
\end{aligned}
$$

If we consider the steady state, (48) with $U=0$ is the 
integral representation of the Poisson equation. Substituting the boundary condition into (48) and considering on the boundary $\bar{C}$, we obtain the integral equation used in the BEM.

\section{Numerical Applications to Two-Dimensional (2D) Problems}

\subsection{Diffusion of Material in Infinite Space without Advection}

For simplicity, we assume the conditions given in (47) and $\sigma=0$. We consider the diffusion of a material placed initially at the center of the coordinates in an infinite region. In other words, the initial condition $C(x, y, 0)=\delta(x, y)$ is specified in the region $-\infty<x<\infty$ and $-\infty<y<\infty$. Using symmetry, we consider only the quarter region given by $0 \leq x \leq L, 0 \leq y \leq B$.

Assuming $\sigma(x, t)=0$ and $U=0$, in this case, we have

$$
\begin{aligned}
& \varepsilon C(x, y, t)-\frac{1}{2 \pi} \int_{0}^{L} C(\xi, 0, t) \frac{y}{(x-\xi)^{2}+y^{2}} d \xi \\
& -\frac{1}{2 \pi} \int_{0}^{B} C(0, \eta, t) \frac{x}{x^{2}+(y-\eta)^{2}} d \eta \\
& =\frac{1}{2 \pi \nu} \int_{0}^{L} \int_{0}^{B} \ln \left(\sqrt{(x-\xi)^{2}+(y-\eta)^{2}}\right) \frac{\partial C(\xi, \eta, t)}{\partial t} d \xi d \eta
\end{aligned}
$$

where $C$ and $\partial C / \partial n$ are assumed to be zero on the boundaries $x=L$ and $y=B$.

For simplicity, we divide the region $0 \leq x \leq L, 0 \leq y \leq B$ into $M \times N$ equal elements with sides $d x$ and $d y$ and denote the center of each element as $\left(x_{i}, y_{j}\right)$, $i=0,1, \cdots, M-1, j=0,1, \cdots, N-1$. In other words, we have

$$
\begin{gathered}
d x=\frac{L}{M}, d y=\frac{B}{N} \\
x_{i}=\frac{d x}{2}+i d x, \quad i=0,1, \cdots, M-1, \\
y_{j}=\frac{d y}{2}+j d y, \quad j=0,1, \cdots, N-1 .
\end{gathered}
$$

We summarize the initial and boundary conditions as follows:

Initial condition:

$$
\begin{aligned}
& C\left(x_{i}, y_{j}, 0\right)=\frac{1}{4 d x d y} \delta_{i 0} \delta_{j 0}, \\
& \quad i=0,1, \cdots, M-1, \quad j=0,1, \cdots, N-1 .
\end{aligned}
$$

Boundary conditions:

$$
\begin{gathered}
\frac{\partial C\left(0, y_{j}, t\right)}{\partial x}=0, \quad j=0,1, \cdots, N-1 \\
\frac{\partial C\left(x_{i}, 0, t\right)}{\partial y}=0, \quad i=0,1, \cdots, M-1 .
\end{gathered}
$$

Equation (49) is then approximated by the following algebraic equation:

$$
\begin{aligned}
& \varepsilon C(x, y, t)-\frac{1}{2 \pi} \sum_{m=0}^{m=M-1} C\left(x_{m}, 0, t\right) \int_{x_{m}-d x / 2}^{x_{m}+d x / 2} \frac{y}{(x-\xi)^{2}+y^{2}} d \xi \\
& -\frac{1}{2 \pi} \sum_{n=0}^{n=N-1} C\left(0, y_{n}, t\right) \int_{y_{n}-d y / 2}^{y_{n}+d y / 2} \frac{x}{x^{2}+(y-\eta)^{2}} d \eta \\
& =\frac{1}{2 \pi \nu} \sum_{m=0}^{m=M-1} \sum_{n=0}^{n=N-1} \frac{\partial C\left(x_{m}, y_{n}, t\right)}{\partial t} \\
& \cdot \int_{x_{m}-d x / 2}^{x_{m}+d x / 2} \int_{y_{n}-d y / 2}^{y_{n}+d y / 2} \ln \left(\sqrt{(x-\xi)^{2}+(y-\eta)^{2}}\right) d \xi d \eta
\end{aligned}
$$

As discussed in Section 3, there are two types of solution: the explicit and implicit solutions. We use the explicit solutions below and consider the two following solutions:

Solution A1.a. Only $\partial C\left(x_{i}, y_{j}, t\right) / \partial t$ at IP is unknown

We approximate the boundary value $C\left(x_{m}, 0, t\right)$ and $C\left(0, y_{n}, t\right)$ as follows:

$$
C\left(x_{m}, 0, t\right) \approx C\left(x_{m}, y_{0}, t\right), C\left(0, y_{n}, t\right) \approx C\left(x_{0}, y_{n}, t\right),
$$

and the algebraic equation is approximated for $i=0,1, \cdots, M-1, j=0,1, \cdots, N-1$ as

$$
\begin{aligned}
& C\left(x_{i}, y_{j}, t\right)-\frac{1}{2 \pi} \sum_{m=0}^{m=M-1} C\left(x_{m}, \eta_{0}, t\right) \int_{x_{m}-d x / 2}^{x_{m}+d x / 2} \frac{y_{j}}{\left(x_{i}-\xi\right)^{2}+y_{j}^{2}} d \xi \\
& -\frac{1}{2 \pi} \sum_{n=0}^{n=N-1} C\left(x_{0}, y_{n}, t\right) \int_{y_{n}-d y / 2}^{y_{n}+d y / 2} \frac{x_{i}}{x_{i}^{2}+\left(y_{j}-\eta\right)^{2}} d \eta \\
& =\frac{1}{2 \pi \nu} \sum_{m=0}^{m=M-1} \sum_{n=0}^{n=N-1} \frac{\partial C\left(x_{m}, y_{n}, t\right)}{\partial t} \\
& \int_{x_{m}-d x / 2}^{x_{m}+d x / 2} \int_{y_{n}-d y / 2}^{y_{n}+d y / 2} \ln \left(\sqrt{\left(x_{i}-\xi\right)^{2}+\left(y_{j}-\eta\right)^{2}}\right) d \xi d \eta
\end{aligned}
$$

The unknowns of this algebraic equation are $\partial C / \partial t$ at IP, and the number of the unknowns is $M \times N$. The number of equations is also $M \times N$. Hence, we can solve this equation. However, the precision is not high because of the approximation in (55).

Solution A1.b. Both $\partial C\left(x_{i}, y_{j}, t\right) / \partial t$ at IP, and $C\left(x_{i}, 0, t\right)$ and $C\left(0, y_{j}, t\right)$ at BP are unknown

For the internal point $\left(x_{i}, y_{j}\right), i=0,1, \cdots, M-1$, $j=0,1, \cdots, N-1$, we have

$$
\begin{aligned}
& C\left(x_{i}, y_{j}, t\right)-\frac{1}{2 \pi} \sum_{m=0}^{m=M-1} C\left(x_{m}, 0, t\right) \int_{x_{m}-d x / 2}^{x_{m}+d x / 2} \frac{y_{j}}{\left(x_{i}-\xi\right)^{2}+y_{j}^{2}} d \xi \\
& -\frac{1}{2 \pi} \sum_{n=0}^{n=N-1} C\left(0, y_{n}, t\right) \int_{y_{n}-d y / 2}^{y_{n}+d y / 2} \frac{x_{i}}{x_{i}^{2}+\left(y_{j}-\eta\right)^{2}} d \eta \\
& =\frac{1}{2 \pi v} \sum_{m=0}^{m=M-1} \sum_{n=0}^{n=N-1} \frac{\partial C\left(x_{m}, y_{n}, t\right)}{\partial t} \\
& \cdot \int_{x_{m}-d x / 2}^{x_{m}+d x / 2} \int_{y_{n}-d y / 2}^{y_{n}+d y / 2} \ln \left(\sqrt{\left(x_{i}-\xi\right)^{2}+\left(y_{j}-\eta\right)^{2}}\right) d \xi d \eta
\end{aligned}
$$

for the boundary point on the $x$-axis $\left(x_{i}, 0\right)$, $i=0,1, \cdots, M-1$, 


$$
\begin{aligned}
& \frac{1}{2} C\left(x_{i}, 0, t\right)-\frac{1}{2 \pi} \sum_{n=0}^{n=N-1} C\left(0, y_{n}, t\right) \int_{y_{n}-d y / 2}^{y_{n}+d y / 2} \frac{x_{i}}{x_{i}^{2}+\eta^{2}} d \eta \\
& =\frac{1}{2 \pi \nu} \sum_{m=0}^{m=M-1} \sum_{n=0}^{n=N-1} \frac{\partial C\left(x_{m}, y_{n}, t\right)}{\partial t} \\
& \int_{x_{m}-d x / 2}^{x_{m}+d x / 2} \int_{y_{n}-d y / 2}^{y_{n}+d y / 2} \ln \left(\sqrt{\left(x_{i}-\xi\right)^{2}+\left(y_{j}-\eta\right)^{2}}\right) d \xi d \eta
\end{aligned}
$$

and for the boundary point on the $y$-axis $\left(0, y_{j}\right)$, $j=0,1, \cdots, N-1$

$$
\begin{aligned}
& \frac{1}{2} C\left(0, y_{j}, t\right)-\frac{1}{2 \pi} \sum_{m=0}^{m=M-1} C\left(x_{m}, 0, t\right) \int_{x_{m}-d x / 2}^{x_{m}+d x / 2} \frac{y_{j}}{\xi^{2}+y_{j}^{2}} d \xi \\
& =\frac{1}{2 \pi v} \sum_{m=0}^{m=M-1} \sum_{n=0}^{m=N-1} \frac{\partial C\left(x_{m}, y_{n}, t\right)}{\partial t} \\
& \cdot \int_{x_{m}-d x / 2}^{x_{m}+d x / 2} \int_{y_{n}-d y / 2}^{y_{n}+d y / 2} \ln \left(\sqrt{\xi^{2}+\left(y_{j}-\eta\right)^{2}}\right) d \xi d \eta
\end{aligned}
$$

The number of unknowns and the number of equations are both $M \times N+M+N$. Thus, we can solve the equation.

The exact solution of this problem is given by

$$
C(x, y, t)=\frac{1}{4 \pi v t} e^{-\frac{x^{2}+y^{2}}{4 v t}}
$$

The numerical results are shown in Fig. 4. The conditions of the calculations are $L=B=1, M=N=20, d t=0.0005$, $t=0.5$, and $\kappa=0.089$. The results indicate that Solution A. $1 \mathrm{~b}$ is more precise than Solution A1.a.

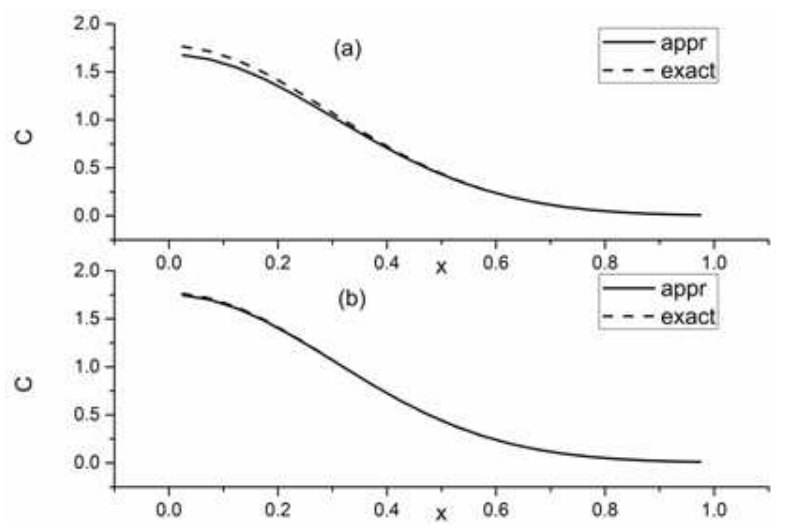

Figure 4. Numerical results at $y=0.025$ ( $t=0.5$ ); (a) Solution A1.a; (b) Solution A1.b.

\subsection{Diffusion of Material in Infinite Space with Advection}

For simplicity, we assume the conditions given in (47) and $\sigma=0$. We assume that the advection velocity $U$ is not zero. In the calculations in Section 5.1, we considered in the region given by $0 \leq x<\infty, 0 \leq y<\infty \quad(0 \leq x<L, 0 \leq y<B$ in computation) using symmetry. However, since this problem is asymmetric, we must consider in the region given by $-\infty<x<\infty,-\infty \leq y<\infty \quad(-L \leq x<L,-B \leq y<B \quad$ in computation). Namely, the size and center of elements are given by

$$
\begin{gathered}
d x=\frac{2 L}{M}, d y=\frac{2 B}{N} \\
x_{i}=-L+(0.5+i) d x, \quad y_{j}=-B+(0.5+j) d y, \\
i=0,1, \cdots, M-1, \quad j=0,1, \cdots, N-1 .
\end{gathered}
$$

The initial and boundary conditions are as follows. Initial condition:

$$
\begin{aligned}
& C\left(x_{i}, 0\right)=\frac{1}{d x d y} \delta_{i M / 2} \delta_{j N / 2}, \\
& \quad i=0,1, \cdots, M-1, \quad j=0,1, \cdots, N-1 .
\end{aligned}
$$

Boundary condition:

$$
\begin{gathered}
C(\mathbf{x}, t)=0, \frac{\partial C(\mathbf{x}, t)}{\partial x}=0 \\
\text { on } \bar{C}
\end{gathered}
$$

$C$ and $\partial C / \partial n$ are assumed to be zero on the boundaries $x= \pm L$ and $y= \pm B$.

We have from (48)

$$
\begin{aligned}
& \varepsilon C(x, y, t)-\frac{U}{2 \pi \kappa} \int_{-L}^{L} \int_{-B}^{B} C(\xi, \eta, t) \frac{(x-\xi)}{(x-\xi)^{2}+(y-\eta)^{2}} d \xi d \eta \\
& =\frac{1}{2 \pi \kappa} \int_{-L}^{L} \int_{-B}^{B} \ln \left(\sqrt{(x-\xi)^{2}+(y-\eta)^{2}}\right) \frac{\partial C(\xi, \eta, t)}{\partial t} d \xi d \eta
\end{aligned}
$$

The exact solution of this problem is given by

$$
C(x, y, t)=\frac{1}{4 \pi \kappa t} e^{-\frac{(x-U t)^{2}+y^{2}}{4 \kappa t}}
$$

We use the explicit Solution A1. The numerical results are shown in Figs. 5 and 6 . The conditions of the calculations are $L=B=2, M=N=41, d t=0.0005, \quad \kappa=0.089$, and $U=1.0$. According to the results, the accuracy of the numerical results is high. The numerical result at $t=1.25$ is affected by the boundary.

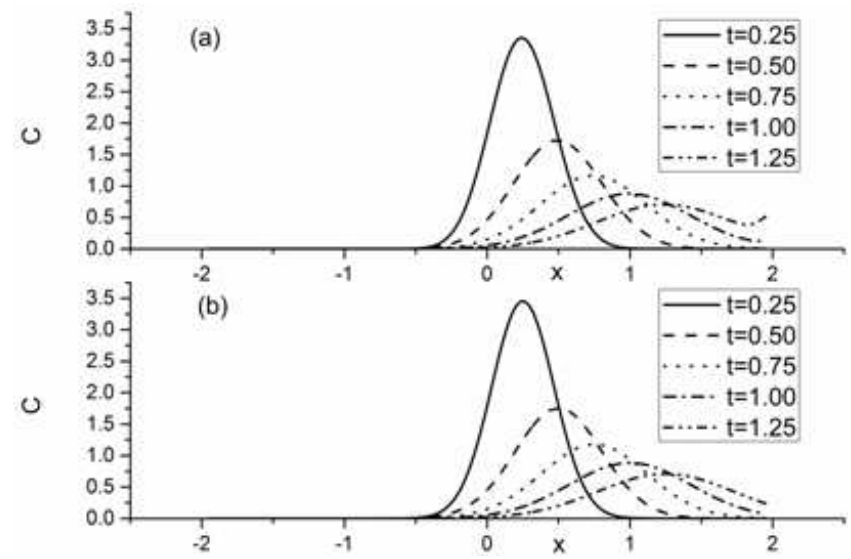

Figure 5. Space distribution of $C$ in case of source type initial value $(y=0)$ : (a) approximate solution; (b) exact solution. 

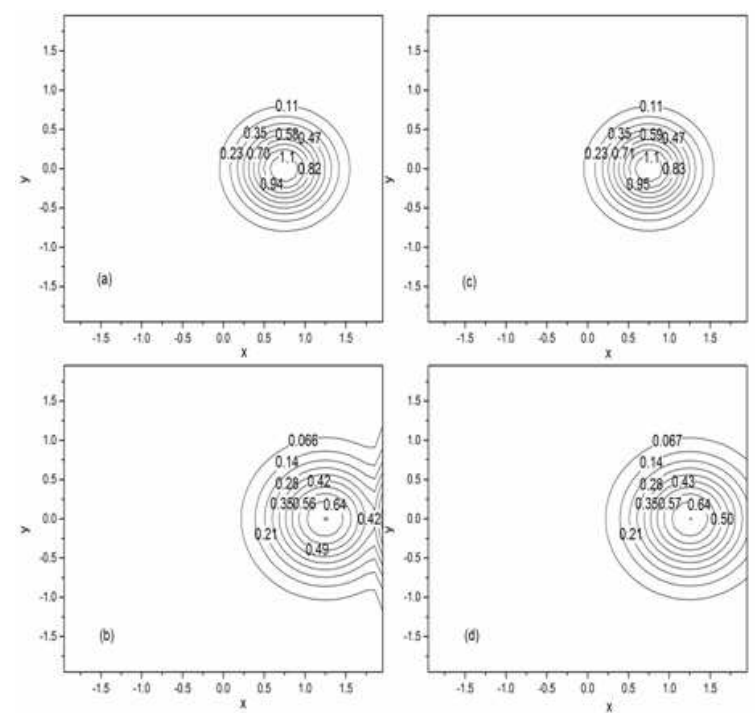

Figure 6. Space distribution of $C$ obtained by approximate and exact solutions: (a) appr., $t=0.75$; (b) appr., $t=1.25$; (c) exact, $t=0.75$; (d) exact, $t=1.25$.

\section{Generalization to Generalized Integral Representation Method (GIRM)}

\subsection{Generalized Integral Representation Method (GIRM) in $1 D$}

We introduce a generalized space fundamental solution $\widetilde{G}(x, \xi)$ defined as

$$
\frac{\partial}{\partial x}\left(\kappa(x, t) \frac{\partial \widetilde{G}(x, \xi)}{\partial x}\right)+u(x, t) \frac{\partial \widetilde{G}(x, \xi)}{\partial x}=\widetilde{\delta}(x, \xi, t)
$$

where $\widetilde{\delta}(x, \xi)$ is derived from $\widetilde{G}(x, \xi)$ when $\widetilde{G}(x, \xi)$ is specified. If we use the Gaussian function:

$$
\widetilde{G}(x, \xi)=\frac{1}{\sqrt{2 \pi} \gamma} \exp \left(-\frac{(x-\xi)^{2}}{2 \gamma^{2}}\right),
$$

then $\widetilde{G}(x, \xi)$ and $\widetilde{\delta}(x, \xi)$ satisfy

$$
\begin{aligned}
\frac{\partial \widetilde{G}(x, \xi)}{\partial x} & =-\frac{(x-\xi)}{\sqrt{2 \pi} \gamma^{3}} \exp \left(-\frac{(x-\xi)^{2}}{2 \gamma^{2}}\right), \\
\frac{\partial^{2} \widetilde{G}(x, \xi)}{\partial x^{2}} & =-\frac{1}{\sqrt{2 \pi} \gamma^{3}} \exp \left(-\frac{(x-\xi)^{2}}{2 \gamma^{2}}\right) \\
& +\frac{(x-\xi)^{2}}{\sqrt{2 \pi} \gamma^{5}} \exp \left(-\frac{(x-\xi)^{2}}{2 \gamma^{2}}\right),
\end{aligned}
$$

and

$$
\begin{aligned}
& \widetilde{\delta}(x, \xi, t)=\frac{\partial}{\partial x}\left(\kappa(x, t) \frac{\partial \widetilde{G}(x, \xi)}{\partial x}\right)+u(x, t) \frac{\partial \widetilde{G}(x, \xi)}{\partial x} \\
& =\frac{\partial \kappa(x, t)}{\partial x} \frac{\partial \widetilde{G}(x, \xi)}{\partial x}+\kappa(x, t) \frac{\partial^{2} \widetilde{G}(x, \xi)}{\partial x^{2}}+u(x, t) \frac{\partial \widetilde{G}(x, \xi)}{\partial x} .
\end{aligned}
$$

Furthermore, when $\widetilde{G}(x, \xi)$ is defined as (66), and $\gamma$ tends to zero, then $\widetilde{G}(x, \xi)$ and $\widetilde{\delta}(x, \xi)$ satisfy

$$
\begin{aligned}
& \tilde{G}(x, \xi) \rightarrow \delta(x, \xi), \\
& \widetilde{\delta}(x, \xi, t) \rightarrow \frac{\partial \kappa(x, t)}{\partial x} \frac{\partial \delta(x, \xi)}{\partial x}+\kappa(x, t) \frac{\partial^{2} \delta(x, \xi)}{\partial x^{2}} \\
&+u(x, t) \frac{\partial \delta(x, \xi)}{\partial x},
\end{aligned}
$$

where $\delta(x, \xi)=\delta(x-\xi)$ is the Dirac delta function:

$$
\delta(x, \xi)=0 \text { for } x \neq \xi \text { and } \int_{-\infty}^{\infty} \delta(x, \xi) d x=1
$$

Assuming (66), $\widetilde{G}(x, \xi)$ is not singular at $x=\xi$ and decreases rapidly as $|x-\xi|$ increases. These properties are useful in the numerical calculations. We can increase the accuracy of the numerical integration and reduce the computation time.

From (1) and (65), we have

$$
\begin{aligned}
0=\int_{0}^{L}\{\widetilde{G}(x, \xi)[ & \frac{\partial}{\partial x}\left(\kappa(x, t) \frac{\partial C(x, t)}{\partial x}\right)-u(x, t) \frac{\partial C(x, t)}{\partial x} \\
& \left.-\left(\frac{\partial C(x, t)}{\partial t}-\sigma(x, t)\right)\right] \\
-C(x, t)[ & {\left[\frac{\partial}{\partial x}\left(\kappa(x, t) \frac{\partial \widetilde{G}(x, \xi)}{\partial x}\right)\right.} \\
& \left.\left.+u(x, t) \frac{\partial \widetilde{G}(x, \xi)}{\partial x}-\widetilde{\delta}(x, \xi, t)\right]\right\} d x .
\end{aligned}
$$

Rewriting (72), we have

$\int_{0}^{L} C(x, t) \tilde{\delta}(x, \xi, t) d x=$

$-\int_{0}^{L}\left[\widetilde{G}(x, \xi) \frac{\partial}{\partial x}\left(\kappa(x, t) \frac{\partial C(x, t)}{\partial x}\right)-C(x, t) \frac{\partial}{\partial x}\left(\kappa(x, t) \frac{\partial \widetilde{G}(x, \xi)}{\partial x}\right)\right] d x$

$+\int_{0}^{L} \tilde{G}(x, \xi)\left(\frac{\partial C(x, t)}{\partial t}-\sigma(x, t)\right) d x+\int_{0}^{L} u(x, t) \frac{\partial \widetilde{G}(x, \xi) C(x, t)}{\partial x} d x$.

Transforming the integral of the first and third terms on the right-hand side of (73) and exchanging $x$ and $\xi$, we derive the generalized integral representation:

$$
\begin{aligned}
\int_{0}^{L} C(\xi, t) \widetilde{\delta}(\xi, x, t) d \xi \\
=-\left[\widetilde{G}(\xi, x) \kappa(\xi, t) \frac{\partial C(\xi, t)}{\partial \xi}-C(\xi, t) \kappa(\xi, t) \frac{\partial \widetilde{G}(\xi, x)}{\partial \xi}\right]_{\xi=0}^{\xi=L} \\
+[u(\xi, t) \widetilde{G}(\xi, x) C(\xi, t)]_{\xi=0}^{\xi=L}-\int_{0}^{L} \frac{\partial u(\xi, t)}{\partial \xi} \widetilde{G}(\xi, x) C(\xi, t) d \xi \\
\quad+\int_{0}^{L} \widetilde{G}(\xi, x)\left(\frac{\partial C(\xi, t)}{\partial t}-\sigma(\xi, t)\right) d \xi .
\end{aligned}
$$

This equation does not include the spatial derivative of the unknown variable $C$ in the integrals.

When $\gamma$ tends to zero, then (69) and (70) make the generalized integral representation, (74), tends to the differential equation, (1), at IP. 
If $C(x, t)$ at IP and $C(x, t)$ or $\partial C(x, t) / \partial x$ at $\mathrm{BP}$ are known from the boundary conditions, then the generalized integral representations are integral equations with $\partial C(x, t) / \partial t$ the unknown variables at IP and $\partial C(x, t) / \partial x$ or $C(x, t)$ the unknown variables at BP. We obtain $C(x, t+d t)$ at IP from $C(x, t+d t)=C(x, t)+d t \partial C(x, t) / \partial t$. If $C(x, 0)$ on IP is known from the initial conditions, then we can solve the initial and boundary value problem using the generalized integral representations. The generalized integral representations are equivalent to the differential equations.

When the diffusion coefficient $\kappa(x, t)$ and the advective velocity $u(x, t)$ are given simply by

$$
\begin{gathered}
\kappa(x, t)=\kappa=\text { const }, \\
u(x, t)=U=\text { const },
\end{gathered}
$$

if we assume (66), (68) and (74) are given by

$$
\begin{aligned}
& \widetilde{\delta}(x, \xi, t) {\left[-\frac{1}{\sqrt{2 \pi} \gamma^{3}} \exp \left(-\frac{(x-\xi)^{2}}{2 \gamma^{2}}\right)+\frac{(x-\xi)^{2}}{\sqrt{2 \pi} \gamma^{5}} \exp \left(-\frac{(x-\xi)^{2}}{2 \gamma^{2}}\right)\right] } \\
&-\frac{(x-\xi)}{\sqrt{2 \pi} \gamma^{3}} U \exp \left(-\frac{(x-\xi)^{2}}{2 \gamma^{2}}\right), \\
& \int_{0}^{L} C(\xi, t) \widetilde{\delta}(\xi, x, t) d \xi \\
&=\int_{0}^{L} \widetilde{G}(\xi, x) \frac{\partial C(\xi, t)}{\partial t} d \xi-\int_{0}^{L} \widetilde{G}(\xi, x) \sigma(\xi, t) d \xi \\
&-\kappa\left[\tilde{G}(L, x) \frac{\partial C(L, t)}{\partial \xi}-C(L, t) \frac{\partial \widetilde{G}(L, x)}{\partial \xi}\right] \\
&+\kappa\left[\tilde{G}(0, x) \frac{\partial C(0, t)}{\partial \xi}-C(0, t) \frac{\partial \widetilde{G}(0, x)}{\partial \xi}\right] \\
&+U[\widetilde{G}(L, x) C(L, t)]-U[\widetilde{G}(0, x) C(0, t)] .
\end{aligned}
$$

\subsection{Diffusion of Material in 1D Infinite Space with Advection}

We discussed the same problem in Section 3.2. For simplicity, we assume (75) and $\sigma=0$. We assume the triangular initial distribution of a material, and the same computation region given by $-L<x<L$ is used. If we assume $L>>1$ and

$$
\frac{\partial C( \pm L, t)}{\partial x}=0 \text { and } C( \pm L, t)=0
$$

the generalized integral representation is, from (77), given by

$$
\int_{-L}^{L} C(\xi, t) \widetilde{\delta}(\xi, x, t) d \xi=\int_{-L}^{L} \tilde{G}(\xi, x) \frac{\partial C(\xi, t)}{\partial t} d \xi
$$

The initial value is specified as

$$
C(x, 0)=\left\{\begin{array}{ll}
\frac{10}{L}\left(x+\frac{L}{10}\right), & \text { if }-\frac{L}{10} \leq x<0 \\
\frac{10}{10}\left(\frac{L}{10}-x\right), & \text { if } 0 \leq x<\frac{L}{10} . \\
0, & \text { otherwise }
\end{array} .\right.
$$

Numerical results are shown in Fig. 7. We used procedure A1 for the numerical calculation. The calculation conditions are $L=4, N=80,160,320, d t=0.001,0.0005,0.00025$, $\kappa=0.01, U=2.0$, and $\gamma=0.16,0.08,0.04$. In the calculations, $N$ or $d x$ was chosen assuming a proper value of the local Peclet number $U d x / \kappa$, and then $d t$ and $\gamma$ were determined keeping the local Courant number $U d t / d x$ and the steepness of the Gaussian function $d x / \gamma$ constant: 0.02 and 0.625 , respectively. If $N$ was increased, then the computational noise was eliminated, and the precise results were obtained.
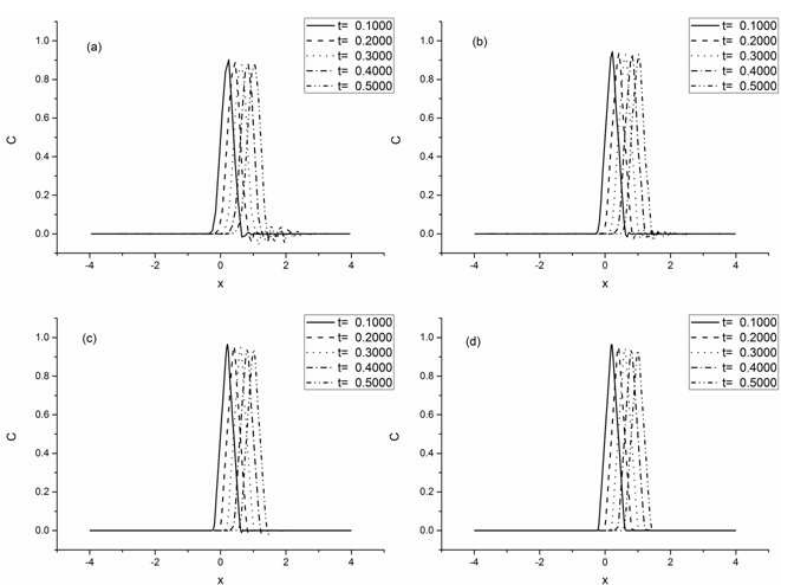

Figiure 7. Space distribution of $C$ in case of triangular initial distribution of a material: (a) approximate solution $(N=80)$; (b) approximate solution ( $N=160)$; (c) approximate solution $(N=320)$; (d) exact solution.

\subsection{Generalized Integral Representation Method (GIRM) in $2 D$}

In the case of $2 \mathrm{D}$, the diffusion equation is given by (34), and we replace (65) by

$$
\nabla \cdot(\kappa(\mathbf{x}, t) \nabla \widetilde{G}(\mathbf{x}, \xi))+(\mathbf{u}(\mathbf{x}, t) \cdot \nabla) \widetilde{G}(\mathbf{x}, \xi)=\widetilde{\delta}(\mathbf{x}, \xi, t) .
$$

The extension of the theory to $3 \mathrm{D}$ is straightforward. We can use the Gaussian function as a generalized space fundamental solution $\widetilde{G}(\mathbf{x}, \xi)$ :

$$
\widetilde{G}(\mathbf{x}, \xi)=\frac{1}{2 \pi \gamma^{2}} \exp \left(-\frac{r^{2}}{2 \gamma^{2}}\right),
$$

where $r=|\mathbf{x}-\xi|=\sqrt{(x-\xi)^{2}+(y-\eta)^{2}}$. Then, we have

$$
\nabla \widetilde{G}(\mathbf{x}, \xi)=-\frac{(\mathbf{x}-\xi)}{2 \pi \gamma^{4}} \exp \left(-\frac{r^{2}}{2 \gamma^{2}}\right)
$$




$$
\nabla^{2} \widetilde{G}(\mathbf{x}, \boldsymbol{\xi})=-\frac{1}{\pi \gamma^{4}} \exp \left(-\frac{r^{2}}{2 \gamma^{2}}\right)+\frac{r^{2}}{2 \pi \gamma^{6}} \exp \left(-\frac{r^{2}}{2 \gamma^{2}}\right)
$$

$$
\begin{aligned}
& \text { and } \\
& \begin{aligned}
\widetilde{\delta} & (\mathbf{x}, \xi, t)=\nabla_{\mathbf{x}} \cdot\left(\kappa(\mathbf{x}, t) \nabla_{\mathbf{x}} \widetilde{G}(\mathbf{x}, \xi)\right)+\left(\mathbf{u} \cdot \nabla_{\mathbf{x}}\right) \widetilde{G}(\mathbf{x}, \xi) \\
\quad & \left(\nabla_{\mathbf{x}} \kappa(\mathbf{x}, t)\right) \cdot\left(\nabla_{\mathbf{x}} \widetilde{G}(\mathbf{x}, \xi)\right)+\kappa(\mathbf{x}, t) \nabla_{\mathbf{x}}^{2} \widetilde{G}(\mathbf{x}, \xi)+\left(\mathbf{u} \cdot \nabla_{\mathbf{x}}\right) \widetilde{G}(\mathbf{x}, \xi)
\end{aligned}
\end{aligned}
$$

When $\widetilde{G}(x, \xi)$ is defined as (82), and $\gamma$ tends to zero, then $\widetilde{G}(\mathbf{x}, \xi)$ satisfies

$$
\widetilde{G}(\mathbf{x}, \xi) \rightarrow \delta(\mathbf{x}, \xi),
$$

where $\delta(\mathbf{x}, \xi)$ is the Dirac delta function in 2D:

$$
\delta(\mathbf{x}, \xi)=\delta(x-\xi) \delta(y-\eta) .
$$

From (34) and (81), we have

$$
\begin{aligned}
0=\iint_{S}\left\{\widetilde { G } ( \mathbf { x } , \xi ) \left[\nabla_{\mathbf{x}} \cdot\left(\kappa(\mathbf{x}, t) \nabla_{\mathbf{x}} C(\mathbf{x}, t)\right)-\left(\mathbf{u}(\mathbf{x}, t) \cdot \nabla_{\mathbf{x}}\right) C(\mathbf{x}, t)\right.\right. \\
\left.-\left(\frac{\partial C(\mathbf{x}, t)}{\partial t}-\sigma(\mathbf{x}, t)\right)\right] \\
-C(\mathbf{x}, t)\left[\nabla_{\mathbf{x}} \cdot\left(\kappa(\mathbf{x}, t) \nabla_{\mathbf{x}} \widetilde{G}(\mathbf{x}, \xi)\right)+\left(\mathbf{u}(\mathbf{x}, t) \cdot \nabla_{\mathbf{x}}\right) \widetilde{G}(\mathbf{x}, \boldsymbol{\xi})\right. \\
-\widetilde{\delta}(\mathbf{x}, \boldsymbol{\xi}, t)]\} d S_{\mathbf{x}} .
\end{aligned}
$$

From (87), we derive the generalized integral representation:

$$
\begin{aligned}
& \iint_{S} C(\xi, t) \widetilde{\delta}(\xi, \mathbf{x}, t) d S_{\xi} \\
& =-\int_{C}\left[\widetilde{G}(\boldsymbol{\xi}, \mathbf{x}) \kappa(\xi, t) \frac{\partial C(\xi, t)}{\partial n_{\xi}}-C(\xi, t) \kappa(\xi, t) \frac{\partial \widetilde{G}(\xi, \mathbf{x})}{\partial n_{\xi}}\right] d l_{\xi} \\
& +\int_{C}[\mathbf{u}(\xi, t) \widetilde{G}(\xi, \mathbf{x}) C(\xi, t)] \cdot \mathbf{n} d l_{\xi}-\iint_{S}\left(\nabla_{\xi} \cdot \mathbf{u}(\xi, t)\right) \widetilde{G}(\xi, \mathbf{x}) C(\xi, t) d S_{\xi} \\
& +\iint_{S} \widetilde{G}(\xi, \mathbf{x})\left(\frac{\partial C(\xi, t)}{\partial t}-\sigma(\xi, t)\right) d S_{\xi} .
\end{aligned}
$$

This equation does not include the spatial derivative of the unknown variable $C$ in the integrals on $S$.

When $\gamma$ tends to zero, then the generalized integral representation, (88), tends to the differential equation given by (34) at IP.

\subsection{Diffusion of Material in 2D Infinite Space with Advection}

We discussed a similar problem in Section 5.2. The computation region is given by $-\infty<x<\infty,-\infty \leq y<\infty$ ( $-L \leq x<L,-B \leq y<B$ in computation). For simplicity, we assume (47) and $\sigma=0$. The generalized integral representation is, from (88), given by

$$
\iint_{S} C(\boldsymbol{\xi}, t) \widetilde{\delta}(\boldsymbol{\xi}, \mathbf{x}, t) d S_{\xi}=\iint_{S} \widetilde{G}(\boldsymbol{\xi}, \mathbf{x}) \frac{\partial C(\boldsymbol{\xi}, t)}{\partial t} d S_{\xi}
$$

The initial value is specified as

$$
C(x, y, 0)=\exp \left(-\frac{1}{2}\left(\frac{8 x}{L}\right)^{2}+\left(\frac{8 y}{B}\right)^{2}\right) .
$$

Numerical results are shown in Fig. 8. We used the explicit procedure A1 for the numerical calculation. The calculation conditions are $L=B=4, \quad M=N=41$, $d t=0.00025, \kappa=0.01, U=1.0$, and $\gamma=0.16$. The accuracy of the numerical results is high.

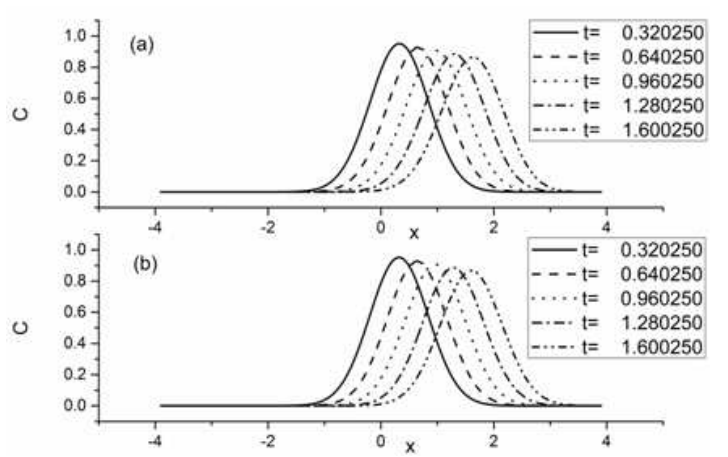

Figure 8. Space distribution of $C$ in case of Gaussian type initial value $(y=0)$ : (a) approximate solution; (b) exact solution.

\section{Conclusions}

An integral representation is obtained using a fundamental solution of a differential-type boundary value problem. If the boundary conditions are substituted into the integral representation, an integral equation is obtained. In the 1D-problems, the diffusion of a material initially concentrated at the center of the space with and without the advection was discussed. The $1 \mathrm{D}$-examples revealed the basic aspects of the integral representation method. Similar problems were also discussed in 2D. As shown in Figs. 1 through 6 , the agreement of the numerical results with the analytical ones was satisfactory. The diffusion and the advection are properly calculated in the examples.

Furthermore, we proposed a generalized integral representation method. The integral representation based on the primary space-differentiation operator is one of the generalized integral representations. In the generalized theory, the fundamental function is chosen first, and the differential equation is defined properly reflecting the fundamental solution and the boundary value problem. In the numerical examples, we used the Gaussian function as the generalized fundamental solution. The stability and the precision of the computation were satisfactory as shown in Figs. 7 and 8. Assuming the Gaussian function as the generalized fundamental solution, we can not only eliminate the singularity but also localize the effect of a point on other points in its neighborhood. These properties are useful in the numerical calculations.

\section{References}

[1] C.A. Brebbia, J.C.F. Telles, L.C. Wrobel, 4.3 Coupled boundary element - Finite difference methods, Boundary Element Techniques, Theory and Applications in Engineering, Springer-Verlag (1984).

[2] B. Nayroles, G. Touzot, P. Villon, Generalizing the finite element method: Diffuse approximation and diffuse elements, Comput. Mechanics 10 (1992) 307-318. 
[3] T. Belytschko, Y.Y. Lu, L. Gu, Element-free Galarkin methods, International Journal for Numerical Methods in Engineering 37 (1994) 229-256.

[4] G. Yagawa, Y. Yamda, Free mesh method: A new meshless finite element method, Computational Mechanics 18 (1996) 383-386.

[5] L.B. Lucy, A numerical approach to the testing of the fission hypotheis, The Astronomical Jounal 82 (12) (1977) 1013-1024.

[6] G.R. Liu, M.B. Liu, Smoothed Particle Hydrodyndmics-a meshfree particle method. World Scientific; ISBN 981-238-456-1 (2003).

[7] H. Isshiki, Discrete differential operators on irregular nodes (DDIN), International Journal for Numerical Methods in Engineering 88 (12) (2011) 1323-1343.

[8] H. Isshiki, Random Collocation Method (RCM), IMECE2010-39054, Vancouver, Canada (2010).

[9] J.S. Uhlman, An integral equation formulation of the equations of motion of an incompressible fluid, NUWC-NPT Technical Report 10,086 15 July (1992). 